\title{
Skin incision versus laser - the influence of different techniques on scar formation and lymphatic drainage in rats. A preliminary report
}

\author{
Norbert Czapla ${ }^{1}$, Katarzyna Grocholewicz², Ewa Sobolewska ${ }^{3}$, Jan Petriczko¹, Piotr Bargiel ${ }^{1}$, Zofia Polakowska ${ }^{4}$, \\ Piotr Prowans ${ }^{1}$
}

1Department of Plastic, Endocrine and General Surgery, Pomeranian Medical University, Szczecin, Poland

${ }^{2}$ Department of Integrated Dentistry, Pomeranian Medical University, Szczecin, Poland

${ }^{3}$ Department of Prosthodontics, Pomeranian Medical University, Szczecin, Poland

${ }^{4}$ Department of Dermatology and Venereology, Pomeranian Medical University, Szczecin, Poland

Adv Dermatol Allergol 2020; XXXVII (2): 269-271

DOI: https://doi.org/10.5114/ada.2019.83521

\begin{abstract}
Introduction: The constantly rising number of skin malignancies and increasing cancer awareness encourage more people to visit outpatient clinics in order to have various skin lesions removed. Despite the fact that scarring is a physiological response to any excision procedure, minimizing the size of it is a goal of every good practitioner. Therefore the question arises whether different techniques used to remove skin lesions may impact the formation and quality of skin scars.

Aim: To perform an evaluation of skin scars formed by laser and surgical incisions and their influence on lymphatic outflow in rats.

Material and methods: Five male rats were used. Using methylene blue, the migration of dye through lymphatic channels of the lower extremity was measured. Afterwards, transverse incisions were made distally using laser and a surgical blade. Wounds were left to heal by secondary intention. After 4 weeks dye migration assessment was repeated and tissue samples were obtained for microscopic evaluation.

Results: Wounds after surgical incisions healed entirely. Wounds after laser treatment had not healed, with a visible area of granulation tissue and hair loss. Significantly worse dye migration was observed in rat extremities after laser therapy than after surgical incision $(p=0.007)$.

Conclusions: The results of the study show that the size of the scar can depend on the incision technique used. Larger scars after laser therapy limit the lymphatic flow of the skin, which may have an adverse effect on mapping sentinel lymph nodes. However, this hypothesis requires further research.
\end{abstract}

Key words: scar, laser, skin cancer, lymphatic drainage.

\section{Introduction}

In recent years, increased occurrence of skin malignancies has been observed. The most common include basal-cell carcinoma, squamous-cell carcinoma and melanoma (which makes up 4\% of all malignant tumors). Special attention should be paid to malignant melanomas, as even a very small primary lesion with a diameter of a few millimeters can already metastasize severely, reducing the overall survival rate $[1,2]$.

Increased skin cancer awareness encourages more people to visit outpatient clinics in order to have a skin lesion removed. Some of them present with an already diagnosed skin malignancy, but a large number of patients would like to have skin lesions excised due to aesthetic indications.

In the material of the Department of Plastic, Endocrine and General Surgery of the Pomeranian Medical University, the percentage of incidental skin cancer and melanoma diagnosis after excision based on aesthetic indications is 10\%. The basic technique used is a classic surgical excision. Laser or cryotherapy is less common. After each procedure a scar forms as a physiological response to injury. Minimizing the size of the skin scar after any procedure is a goal of every practitioner regardless of

\footnotetext{
Address for correspondence: Jan Petriczko MD, PhD, Department of Plastic, Endocrine and General Surgery, Pomeranian Medical University, Szczecin, Poland, phone: +48694442 577, e-mail: jan.petriczko@gmail.com, jan.petriczko@pum.edu.pl Received: 23.01.2019, accepted: 11.02.2019.
} 
the technique used. Therefore the question arises whether the belief among surgeons that the size of the scar depends on the excision technique can be confirmed by an objective evaluation of the postoperative area.

\section{Aim}

The aim of this study was to perform an evaluation of skin scars formed by laser and surgical incisions and their influence on lymphatic outflow in rats.

\section{Material and methods}

Local Ethics Committee approval was obtained prior to the beginning of the study, and each researcher involved in contact with animals had the requisite training and certificates. For this experiment five male rats, $\mathrm{Wi}$ star breed, were used. Each weighed about 250-300 g. The animals were anaesthetized with subcutaneous ketamine injections in the dose of $100 \mathrm{mg} / \mathrm{kg}$ of body weight. After anaesthesia the medial aspects of their thighs were shaved. Next, $0.02 \mathrm{~cm}^{2}$ of methylene blue dye was injected with a $1 \mathrm{ml}$ syringe subcutaneously to form $5 \mathrm{~mm}$ vesicles. The injections were located on the inner thighs at the level of the knee joints. After 30 min the migration of the dye was assessed by measuring the length of dyed skin (in $\mathrm{mm}$ ) from the border of the vesicle towards the inguinal area (Table 1). After 7 days and elimination of the dye, transverse incisions with a surgical blade were made on the inner surface of the right thigh at about half its length. On the left thigh similar incisions were made with a KaVo Master-laser diode (power 0.2-8 W, peak power: $10 \mathrm{~W}$, wavelength

Table 1. Range of penetration of the dye in healthy skin and skin scar after $30 \mathrm{~min}$

\begin{tabular}{cccc}
\hline No. & $\begin{array}{c}\text { Dye penetration } \\
\text { in healthy skin } \\
{[\mathrm{mm}]}\end{array}$ & $\begin{array}{c}\text { Dye penetration } \\
\text { through surgical } \\
\text { incision scar [mm] }\end{array}$ & $\begin{array}{c}\text { Dye penetration } \\
\text { through laser } \\
\text { scar [mm] }\end{array}$ \\
\hline 1 & 15 & 9 & 3 \\
\hline 2 & 11 & 13 & 2 \\
\hline 3 & 13 & 4 & 0 \\
\hline 4 & 12 & 27 & 17 \\
\hline 5 & 30 & 14 & 0 \\
\hline
\end{tabular}

$890 \mathrm{~nm})$. Wounds were left to heal by secondary intention. All animals were placed in cages, ensuring peace and food without limits. After 4 weeks wound healing was assessed. Animals were once again anaesthetized with ketamine and received injections with $0.02 \mathrm{~cm}^{3}$ of methylene blue. The dye was injected distally from the previous skin incisions. After 30 min the migration of dye was measured. Tissue samples from the scar were obtained for microscopic examination.

\section{Statistical analysis}

Statistical analysis was performed with the $\chi^{2}$ test.

\section{Results}

Wounds after surgical incisions healed entirely, leaving a thin and practically invisible scar. Rat number 3 presented with a slightly wider scar, without fur. Wounds after laser treatment had not healed. In rats 2, 3, 4, 5 in the area of laser incision 1-1.5 mm fields of granulation tissue without fur were observed. In rat number 1, 80\% of wound length had healed with visible epidermis and hair loss on both poles of the scar.

When comparing the results, the dye penetration in healthy skin and the surgical scar did not differ $(p=0.6)$, but a statistically significant difference was found between the penetration through healthy skin and the laser scar $(p=0.038)$. Moreover, significantly worse dye migration was observed after laser therapy than surgical incision $(p=0.007)$ when comparing each rat individually (Table 2).

\section{Discussion}

The principle of laser therapy is removing skin tissue by thermal energy and ablation. Authors have described damage of skin tissue beyond the zone of laser activity. Its extent depends on many parameters such as power, wavelength and time of exposure. Baleg et al. found in a rat model that the amount of damaged tissues using $\mathrm{CO}_{2}$ laser with the wavelength of $600 \mathrm{~nm}$ increased as the epithelium was removed [3]. Omi et al. observed a difference in the depth of skin damage using fractional ablative and non-ablative laser where the zone of skin damage was about $300 \mu \mathrm{m}$ [4]. Walsh et al. also found in a guinea pig model that removing skin tissue with laser creates a damage zone [5]. All authors agree that the ad-

Table 2. Microscopic evaluation of excised scar samples

\begin{tabular}{lcccc}
\hline No. & Epidermis - surgical incision & Epidermis - laser & Dermis - surgical incision & Dermis - laser \\
\hline 1 & No change & Ulceration & No fibrosis & Fibrosis,++ \\
\hline 2 & No change & Ulceration & No fibrosis & Fibrosis +++ subcutaneous tissue coagulated \\
\hline 3 & Ulceration & Ulceration & Fibrosis +++ & Fibrosis +++ \\
\hline 4 & No change & Ulceration & Fibrosis + & Fibrosis +++ subcutaneous tissue coagulated \\
\hline 5 & No change & Ulceration & No fibrosis & Fibrosis +++ subcutaneous tissue coagulated \\
\hline
\end{tabular}


equate choice of laser parameters minimizes unwanted tissue damage. However, the popularity of laser therapy is not always matched by the clinician's expertise. Observations show that the learning curve for classical surgical techniques is steeper. It seems that learning laser treatment requires more time and practice.

The performed study showed that damage to the skin and subcutaneous tissue caused by laser treatment was greater than that caused by surgical incision. The healing time after laser treatment was longer and exceeded 4 weeks in rats. Microscopic scar analysis showed that the fibrosis and coagulation zone was larger after laser treatment than after a surgical incision. Clinical observations on patients show that the healing time after laser therapy is longer than when using surgical techniques. It is known that prolonged healing and associated inflammation can cause a bigger scar. Many authors have studied the ablation zone after laser treatment, but the role of the scars on lymphatic flow in the skin has never been studied before [6]. The size of a scar after a surgical procedure could have significant clinical implications in case of a postoperative melanoma diagnosis and the necessity of sentinel lymph node mapping. Another question arises whether the cutaneous lymphatic flow insufficiency could have an effect on sentinel lymph node mapping. Lopez-Prior demonstrated in a clinical trial that previous breast surgery (including aesthetic) did not have a significant effect on mapping the sentinel lymph node [7]. No papers on the correlation of postoperative or post-traumatic scars in limb sentinel lymph node mapping were found. It appears that clinical situations where a scar located on the lower or upper extremity blocks the path of lymphatic outflow are rare, but there is no evidence that in such cases the accuracy of lymphatic node mapping would not be altered, thus leading to false negative results. The research performed on a rat model showed that a deep scar can interfere with the lymphatic flow in the skin. It must be noted that deeper scars after laser therapy can be a result not only of the device parameters, but also of lesser experience. Therefore when excising potentially malignant skin lesions it is advised to use the technique the clinician is most proficient at.

\section{Conclusions}

The results of the study show that the size of the scar can depend on the incision technique used. Larger scars after laser therapy limit the lymphatic flow of the skin, which may have an adverse effect on mapping sentinel lymph nodes. However, this hypothesis requires further research.

\section{Conflict of interest}

The authors declare no conflict of interest.

\section{References}

1. Valsamaki P, Zanglis A, Gerali S. Cutaneous malignant melanoma: diagnostic procedures and their evaluation in diagnosing and mapping sentinel nodes. Hell J Nuci 2009; 12: 296-303.

2. van der Ploeg IM, Madu MF, van der Hage JA, et al. Blue dye can be safely omitted in most sentinel node procedures for melanoma. Melanoma Res 2016; 26: 464-8.

3. Baleg SM, Bidin N, Suan LP, et al. The effect of CO2 laser treatment on skin tissue. J Cosmet Dermatol 2015; 14: 246-53.

4. Omi T, Numano K. The role of $\mathrm{CO} 2$ laser and fractional $\mathrm{CO} 2$ laser in dermatology. Laser Therapy 2014; 23: 49-60.

5. Walsh JT Jr, Flotte TJ, Anderson RR, Deutsch TF. Pulsed CO2 laser tissue ablation: effect of tissue type and pulse duration on thermal damage. Lasers Surg Med 1988; 8: 108-18.

6. Haedersdal M. Cutaneous side effects from laser treatment of the skin: skin cancer, scars, wounds, pigmentary changes, and purpura: use of pulsed dye laser, copper vapor laser, and argon laser. Acta Derm Venereol Suppl (Stockh) 1999; 207: 1-32.

7. López-Prior V, Díaz-Expósito R, Casáns Tormo I. Influence of previous breast surgery in sentinel lymph node biopsy in patients with breast cancer. Rev Esp Med Nucl Imagen Mol 2017; 36: 212-8. 